\title{
ON POMPEIU-ČEBYŠEV TYPE INEQUALITIES FOR POSITIVE LINEAR MAPS OF SELFADJOINT OPERATORS IN INNER PRODUCT SPACES
}

\author{
MOHAMMAD W. ALOMARI
}

\begin{abstract}
In this work, generalizations of some inequalities for continuous $h$ synchronous ( $h$-asynchronous) functions of linear bounded selfadjoint operators under positive linear maps in Hilbert spaces are proved.
\end{abstract}

\section{INTRODUCTION}

Let $\mathcal{B}(H)$ be the Banach algebra of all bounded linear operators defined on a complex Hilbert space $(H ;\langle\cdot, \cdot\rangle)$ with the identity operator $1_{H}$ in $\mathcal{B}(H)$. Let $A \in \mathcal{B}(H)$ be a selfadjoint linear operator on $(H ;\langle\cdot, \cdot\rangle)$. Let $C(\operatorname{sp}(A))$ be the set of all continuous functions defined on the spectrum of $A(\operatorname{sp}(A))$ and let $C^{*}(A)$ be the $C^{*}$-algebra generated by $A$ and the identity operator $1_{H}$.

Let us define the map $\mathcal{G}: C(\operatorname{sp}(A)) \rightarrow C^{*}(A)$ with the following properties ([5], p.3):

(1) $\mathcal{G}(\alpha f+\beta g)=\alpha \mathcal{G}(f)+\beta \mathcal{G}(g)$, for all scalars $\alpha, \beta$.

(2) $\mathcal{G}(f g)=\mathcal{G}(f) \mathcal{G}(g)$ and $\mathcal{G}(\bar{f})=\mathcal{G}(f)^{*}$; where $\bar{f}$ denotes to the conjugate of $f$ and $\mathcal{G}(f)^{*}$ denotes to the Hermitian of $\mathcal{G}(f)$.

(3) $\|\mathcal{G}(f)\|=\|f\|=\sup _{t \in \operatorname{sp}(A)}|f(t)|$.

(4) $\mathcal{G}\left(f_{0}\right)=1_{H}$ and $\mathcal{G}\left(f_{1}\right)=A$, where $f_{0}(t)=1$ and $f_{1}(t)=t$ for all $t \in \operatorname{sp}(A)$.

Accordingly, we define the continuous functional calculus for a selfadjoint operator $A$ by

$$
f(A)=\mathcal{G}(f) \text { for all } f \in C(\operatorname{sp}(A)) .
$$

If both $f$ and $g$ are real valued functions on $\operatorname{sp}(A)$ then the following important property holds:

$$
f(t) \geq g(t) \text { for all } t \in \operatorname{sp}(A) \text { implies } f(A) \geq g(A),
$$

in the operator order of $\mathcal{B}(H)$.

In [1] and formally in [2], the author of this paper generalized the concept of monotonicity as follows:

Date: Received: xxxxxx; Revised: yyyyyy; Accepted: zzzzzz.

2010 Mathematics Subject Classification. Primary 47A63; Secondary 47A99.

Key words and phrases. Hilbert space, Selfadjoint operators, $h$-Synchronization.

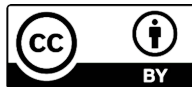


Definition 1.1. A real valued function $f$ defined on $[a, b]$ is said to be increasing (decreasing) with respect to a positive function $h:[a, b] \rightarrow \mathbb{R}_{+}$or simply $h_{\text {- }}$ increasing ( $h$-decreasing) if and only if

$$
h(x) f(t)-h(t) f(x) \geq(\leq) 0,
$$

whenever $t \geq x$ for every $x, t \in[a, b]$. In special case if $h(x)=1$ we refer to the original monotonicity. Accordingly, for $0<a<b$ we say that $f$ is $t^{r}$-increasing $\left(t^{r}\right.$-decreasing) for $r \in \mathbb{R}$ if and only if

$$
x \leq t \Longrightarrow x^{r} f(t)-t^{r} f(x) \geq(\leq) 0
$$

for every $x, t \in[a, b]$.

Example 1.2. Let $0<a<b$ and define $f:[a, b] \rightarrow \mathbb{R}$ given by

(1) $f(s)=1$, then $f$ is $t^{r}$-decreasing for all $r>0$ and $t^{r}$-increasing for all $r<0$.

(2) $f(s)=s$, then $f$ is $t^{r}$-decreasing for all $r>1$ and $t^{r}$-increasing for all $r<1$.

(3) $f(s)=s^{-1}$, then $f$ is $t^{r}$-decreasing for all $r>-1$ and $t^{r}$-increasing for all $r<-1$.

Remark 1.3. Every $h$-increasing function is increasing. The converse need not be true. For more details see [2].

The concept of synchronization has a wide range of usage in several areas of mathematics. Simply, two functions $f, g:[a, b] \rightarrow \mathbb{R}$ are called synchronous (asynchronous) if and only if the inequality

$$
(f(t)-f(x))(g(t)-g(x)) \geq(\leq) 0,
$$

holds for all $x, t \in[a, b]$.

In [2], Alomari generalized the concept of synchronization of functions of real variables. Indeed, we have

Definition 1.4. The real valued functions $f, g:[a, b] \rightarrow \mathbb{R}$ are called synchronous (asynchronous) with respect to a non-negative function $h:[a, b] \rightarrow \mathbb{R}_{+}$or simply $h$-synchronous ( $h$-asynchronous) if and only if

$$
(h(y) f(x)-h(x) f(y))(h(y) g(x)-h(x) g(y)) \geq(\leq) 0
$$

for all $x, y \in[a, b]$.

In other words if both $f$ and $g$ are either $h$-increasing or $h$-decreasing then

$$
(h(y) f(x)-h(x) f(y))(h(y) g(x)-h(x) g(y)) \geq 0 .
$$

While, if one of the function is $h$-increasing and the other is $h$-decreasing then

$$
(h(y) f(x)-h(x) f(y))(h(y) g(x)-h(x) g(y)) \leq 0 .
$$

In special case if $h(x)=1$ we refer to the original synchronization. Accordingly, for $0<a<b$ we say that $f$ and $g$ are $t^{r}$-synchronous ( $t^{r}$-asynchronous) for $r \in \mathbb{R}$ if and only if

$$
\left(x^{r} f(t)-t^{r} f(x)\right)\left(x^{r} g(t)-t^{r} g(x)\right) \geq(\leq) 0
$$

for every $x, t \in[a, b]$. 
Remark 1.5. In Definition (1.4), if $f=g$ then $f$ and $g$ are always $h$-synchronous regardless of $h$-monotonicity of $f$ (or $g$ ). In other words, a function $f$ is always $h$-synchronous with itself.

Example 1.6. Let $0<a<b$ and define $f, g:[a, b] \rightarrow \mathbb{R}$ given by

(1) $f(s)=1=g(s)$, then $f$ and $g$ are $t^{r}$-synchronous for all $r \in \mathbb{R}$.

(2) $f(s)=1$ and $g(s)=s$, then $f$ is $t^{r}$-synchronous for all $r \in(-\infty, 0) \cup(1, \infty)$ and $t^{r}$-asynchronous for all $0<r<1$.

(3) $f(s)=1$ and $g(s)=s^{-1}$, then $f$ is $t^{r}$-synchronous for all $r \in(-\infty,-1) \cup$ $(0, \infty)$ and $t^{r}$-asynchronous for all $-1<r<0$.

(4) $f(s)=s$ and $g(s)=s^{-1}$, then $f$ is $t^{r}$-synchronous for all $r \in(-\infty,-1) \cup$ $(1, \infty)$ and $t^{r}$-asynchronous for all $-1<r<1$.

In [3], Dragomir studied the Čebyšev functional

$$
C(f, g ; A, x):=\langle f(A) g(A) x, x\rangle-\langle g(A) x, x\rangle\langle f(A) x, x\rangle,
$$

for any selfadjoint operator $A \in \mathcal{B}(H)$ and $x \in H$ with $\|x\|=1$.

In [3], proved the following result concerning continuous synchronous (asynchronous) functions of selfadjoint linear operators in Hilbert spaces.

Theorem 1.7. Let $A$ be a selfadjoint operator with $\operatorname{sp}(A) \subset[\gamma, \Gamma]$ for some real numbers $\gamma, \Gamma$ with $\gamma<\Gamma$. If $f, g:[\gamma, \Gamma] \rightarrow \mathbb{R}$ are continuous and synchronous (asynchronous) on $[\gamma, \Gamma]$, then

$$
\langle f(A) g(A) x, x\rangle \geq(\leq)\langle g(A) x, x\rangle\langle f(A) x, x\rangle
$$

for any $x \in H$ with $\|x\|=1$.

In [2], Alomari generalized Theorem 1.7 for continuous $h$-synchronous $(h$ asynchronous) functions of selfadjoint linear operators in Hilbert spaces by introduciing the Pompeiu-Čebyšev functional such as:

$$
\begin{aligned}
\mathcal{P}(f, g, h ; A, x):=\left\langle h^{2}(A) x, x\right\rangle\langle f(A) g(A) x, x\rangle & \\
& -\langle h(A) g(A) x, x\rangle\langle h(A) f(A) x, x\rangle
\end{aligned}
$$

for $x \in H$ with $\|x\|=1$. This naturally, generalizes the Čebyšev functional (1.3).

Moreover, he proved the following essential result:

Theorem 1.8. Let $A$ be a selfadjoint operator with $\operatorname{sp}(A) \subset[\gamma, \Gamma]$ for some real numbers $\gamma, \Gamma$ with $\gamma<\Gamma$. Let $h:[\gamma, \Gamma] \rightarrow \mathbb{R}_{+}$be a non-negative and continuous function. If $f, g:[\gamma, \Gamma] \rightarrow \mathbb{R}$ are continuous and both $f$ and $g$ are $h$-synchronous (h-asynchronous) on $[\gamma, \Gamma]$, then

$$
\left\langle h^{2}(A) x, x\right\rangle\langle f(A) g(A) x, x\rangle \geq(\leq)\langle h(A) g(A) x, x\rangle\langle h(A) f(A) x, x\rangle
$$

for any $x \in H$ with $\|x\|=1$.

For more related results, we refer the reader to [4], [6] and [7].

In this work, some inequalities for continuous $h$-synchronous ( $h$-asynchronous) functions of linear bounded selfadjoint operators under positive linear maps in Hilbert spaces of the Pompeiu-Cebyšev functional (1.5) are proved. The proof Techniques are similar to that ones used in [4]. 


\section{Main Results}

Let us start with the following result regarding the positivity of $\mathcal{P}(f, g, h ; A, x)$.

Theorem 2.1. Let $A$ be a selfadjoint operator with $\operatorname{sp}(A) \subset[\gamma, \Gamma]$ for some real numbers $\gamma, \Gamma$ with $\gamma<\Gamma$. Let $h:[\gamma, \Gamma] \rightarrow \mathbb{R}_{+}$be a non-negative and continuous function. If $f, g:[\gamma, \Gamma] \rightarrow \mathbb{R}$ are continuous and both $f$ and $g$ are $h$-synchronous (h-asynchronous) on $[\gamma, \Gamma]$, then

$$
\begin{aligned}
\left\langle\phi\left(h^{2}(B)\right) y, y\right\rangle \cdot & \langle\varphi(f(A) g(A)) x, x\rangle \\
& +\left\langle\varphi\left(h^{2}(A)\right) x, x\right\rangle \cdot\langle\phi(f(B) g(B)) y, y\rangle \\
\geq & \langle\varphi(h(A) f(A)) x, x\rangle \cdot\langle\phi(h(B) g(B)) y, y\rangle \\
& \quad+\langle\varphi(h(A) g(A)) x, x\rangle \cdot\langle\phi(h(B) f(B)) y, y\rangle
\end{aligned}
$$

for each $x, y \in H$ with $\|x\|=\|y\|=1$.

$$
\begin{aligned}
\left\langle\phi\left(h^{2}(A)\right) y, y\right\rangle \cdot & \langle\varphi(f(A) g(A)) x, x\rangle \\
& +\left\langle\varphi\left(h^{2}(A)\right) x, x\right\rangle \cdot\langle\phi(f(A) g(A)) y, y\rangle \\
\geq(\leq)\langle\varphi(h(A) f(A)) x, x\rangle \cdot\langle\phi(h(A) g(A)) y, y\rangle & \quad+\langle\varphi(h(A) g(A)) x, x\rangle \cdot\langle\phi(h(A) f(A)) y, y\rangle
\end{aligned}
$$

for each $x \in H$ with $\| x=1$.

Proof. Since $f$ and $g$ are $h$-synchronous then

$$
(h(s) f(t)-h(t) f(s))(h(s) g(t)-h(t) g(s)) \geq 0,
$$

and this is allow us to write

$$
\begin{aligned}
h^{2}(s) f(t) g(t)+h^{2}(t) f(s) & g(s) \\
& \geq h(s) h(t) f(t) g(s)+h(s) h(t) g(t) f(s)
\end{aligned}
$$

for all $t, s \in[a, b]$. We fix $s \in[a, b]$ and apply the functional calculus; property (1.1) for inequality (2.3) for the operator $A$, then we have for each $x \in H$ with $\|x\|=1$, that

$$
\begin{aligned}
h^{2}(s) 1_{H} \cdot f(A) g(A) & +h^{2}(A) \cdot f(s) g(s) 1_{H} \\
& \geq h(A) f(A) \cdot h(s) g(s) 1_{H}+h(A) g(A) \cdot h(s) f(s) 1_{H},
\end{aligned}
$$

and since $\varphi$ is normalized positive linear map we get

$$
\begin{aligned}
h^{2}(s) 1_{H} \cdot \varphi & (f(A) g(A))+\varphi\left(h^{2}(A)\right) \cdot f(s) g(s) 1_{H} \\
& \geq \varphi(h(A) f(A)) \cdot h(s) g(s) 1_{H}+\varphi(h(A) g(A)) \cdot h(s) f(s) 1_{H},
\end{aligned}
$$

and this is equivalent to write

$$
\begin{aligned}
& h^{2}(s) 1_{H} \cdot\langle\varphi(f(A) g(A)) x, x\rangle+\left\langle\varphi\left(h^{2}(A)\right) x, x\right\rangle \cdot f(s) g(s) 1_{H} \\
& \quad \geq\langle\varphi(h(A) f(A)) x, x\rangle \cdot h(s) g(s) 1_{H}+\langle\varphi(h(A) g(A)) x, x\rangle \cdot h(s) f(s) 1_{H},
\end{aligned}
$$


Applying property (1.1) again for inequality (2.4) but for the operator $B$, then we have for each $y \in H$ with $\|y\|=1$, that

$$
\begin{aligned}
& h^{2}(B) \cdot\langle\varphi(f(A) g(A)) x, x\rangle+\left\langle\varphi\left(h^{2}(A)\right) x, x\right\rangle \cdot f(B) g(B) \\
& \quad \geq\langle\varphi(h(A) f(A)) x, x\rangle \cdot h(B) g(B)+\langle\varphi(h(A) g(A)) x, x\rangle \cdot h(B) f(B),
\end{aligned}
$$

and since $\phi$ is normalized positive linear map we get

$$
\begin{aligned}
& \left\langle\phi\left(h^{2}(B)\right) y, y\right\rangle \cdot\langle\varphi(f(A) g(A)) x, x\rangle+\left\langle\varphi\left(h^{2}(A)\right) x, x\right\rangle \cdot\langle\phi(f(B) g(B)) y, y\rangle \\
\geq & \langle\varphi(h(A) f(A)) x, x\rangle \cdot\langle\phi(h(B) g(B)) y, y\rangle+\langle\varphi(h(A) g(A)) x, x\rangle \cdot\langle\phi(h(B) f(B)) y, y\rangle,
\end{aligned}
$$

for each $x, y \in H$ with $\|x\|=\|y\|=1$, which gives the required results in (2.1). To obtain (2.2) we set $B=A$ in (2.1). The revers case follows trivially, and this completes the proof.

Corollary 2.2. Let $A$ be a selfadjoint operator with $\operatorname{sp}(A) \subset[\gamma, \Gamma]$ for some real numbers $\gamma, \Gamma$ with $\gamma<\Gamma$. Let $h:[\gamma, \Gamma] \rightarrow \mathbb{R}_{+}$be a non-negative and continuous function. If $f, g:[\gamma, \Gamma] \rightarrow \mathbb{R}$ are continuous and both $f$ and $g$ are synchronous ( asynchronous) on $[\gamma, \Gamma]$, then

$$
\begin{aligned}
& \langle\varphi(f(A) g(A)) x, x\rangle+\langle\phi(f(B) g(B)) y, y\rangle \\
& \quad \geq(\leq)\langle\varphi(f(A)) x, x\rangle\langle\phi(g(B)) y, y\rangle+\langle\varphi(g(A)) x, x\rangle\langle\phi(f(B)) y, y\rangle
\end{aligned}
$$

for each $x, y \in H$ with $\|x\|=\|y\|=1$. In special case, the following Čebyšev inequality for positive linear maps of selfadjoint operator is valid

$$
\begin{aligned}
& \langle\varphi(f(A) g(A)) x, x\rangle+\langle\varphi(f(A) g(A)) x, x\rangle \\
& \quad \geq(\leq)\langle\varphi(f(A)) x, x\rangle\langle\varphi(g(A)) x, x\rangle+\langle\varphi(g(A)) x, x\rangle\langle\varphi(f(A)) x, x\rangle
\end{aligned}
$$

for each $x \in H$ with $\|x\|=1$.

Proof. Setting $h(t)=1$ in both (2.1) and (2.2). Also, in (2.2) take $\phi=\varphi, B=A$ and $y=x$.

Remark 2.3. Setting $\phi=\varphi, B=A$ and $y=x$ in (2.1), we get

$$
\begin{aligned}
\left\langle\varphi\left(h^{2}(A)\right) x, x\right\rangle & \cdot\langle\varphi(f(A) g(A)) x, x\rangle \\
& +\left\langle\varphi\left(h^{2}(A)\right) x, x\right\rangle \cdot\langle\varphi(f(A) g(A)) x, x\rangle \\
\geq(\leq)\langle\varphi(h(A) f(A)) x, x\rangle \cdot\langle\varphi(h(A) g(A)) x, x\rangle & +\langle\varphi(h(A) g(A)) x, x\rangle \cdot\langle\varphi(h(A) f(A)) x, x\rangle
\end{aligned}
$$

for each $x \in H$ with $\|x\|=1$.

The following generalization of Cauchy-Schwarz inequality holds.

Corollary 2.4. Let $A$ be a selfadjoint operator with $\operatorname{sp}(A) \subset[\gamma, \Gamma]$ for some real numbers $\gamma, \Gamma$ with $\gamma<\Gamma$. Let $h:[\gamma, \Gamma] \rightarrow \mathbb{R}_{+}$be a non-negative and continuous 
function. If $f:[\gamma, \Gamma] \rightarrow \mathbb{R}$ is continuous and h-synchronous on $[\gamma, \Gamma]$, then

$$
\begin{array}{r}
\left\langle\phi\left(h^{2}(B)\right) y, y\right\rangle \cdot\left\langle\varphi\left(f^{2}(A)\right) x, x\right\rangle+\left\langle\varphi\left(h^{2}(A)\right) x, x\right\rangle \cdot\left\langle\phi\left(f^{2}(B)\right) y, y\right\rangle \\
\geq 2\langle\varphi(h(A) f(A)) x, x\rangle \cdot\langle\phi(h(B) f(B)) y, y\rangle
\end{array}
$$

for each $x, y \in H$ with $\|x\|=\|y\|=1$. In particular, we have

$$
\left\langle\varphi\left(h^{2}(A)\right) x, x\right\rangle \cdot\left\langle\varphi\left(f^{2}(A)\right) x, x\right\rangle \geq\langle\varphi(h(A) f(A)) x, x\rangle^{2}
$$

for each $x \in H$ with $\|x\|=1$.

Proof. Setting $f=g$ in both (2.1) and (2.2). Also, in (2.2) take $\phi=\varphi, B=A$ and $y=x$, so that the desired results hold.

Corollary 2.5. Let $A$ be a selfadjoint operator with $\operatorname{sp}(A) \subset[\gamma, \Gamma]$ for some real numbers $\gamma, \Gamma$ with $0<\gamma<\Gamma$. If $f, g:[\gamma, \Gamma] \rightarrow \mathbb{R}$ are continuous and $t$-synchronous (t-asynchronous) on $[\gamma, \Gamma]$, then

$$
\begin{array}{r}
\left\langle\phi\left(B^{2}\right) y, y\right\rangle \cdot\langle\varphi(f(A) g(A)) x, x\rangle+\left\langle\varphi\left(A^{2}\right) x, x\right\rangle \cdot\langle\phi(f(B) g(B)) y, y\rangle \\
\geq(\leq)\langle\varphi(A f(A)) x, x\rangle \cdot\langle\phi(B g(B)) y, y\rangle \\
+\langle\varphi(A g(A)) x, x\rangle \cdot\langle\phi(B f(B)) y, y\rangle
\end{array}
$$

for each $x, y \in H$ with $\|x\|=\|y\|=1$.

Proof. Setting $h(t)=t$ in (2.1) we get the desired result.

Before we state our next remark, we interested to give the following example.

Example 2.6. (1) If $f(s)=s^{p}$ and $g(s)=s^{q}(s>0)$, then $f$ and $g$ are $t^{r}$ synchronous for all $p, q>r>0$ and $t^{r}$-asynchronous for all $p>r>q>0$.

(2) If $f(s)=s^{p}$ and $g(s)=\log (s)(s>1)$, then $f$ is $t^{r}$-synchronous for all $p<r<0$ and $t^{r}$-asynchronous for all $r<p<0$.

(3) If $f(s)=\exp (s)=g(s)$, then $f$ is $t^{r}$-synchronous for all for all $r \in \mathbb{R}$.

Remark 2.7. Using Example 2.6 we can observe the following special cases:

(1) If $f(s)=s^{p}$ and $g(s)=s^{q}(s>0)$, then $f$ and $g$ are $t^{r}$-synchronous for all $p, q>r>0$, so that we have

$$
\begin{aligned}
\left\langle\phi\left(B^{2 r}\right) y, y\right\rangle\left\langle\varphi\left(A^{p+q}\right) x, x\right\rangle+\left\langle\varphi\left(A^{2 r}\right) x, x\right\rangle\left\langle\phi\left(B^{p+q}\right) y, y\right\rangle & \\
& \geq\left\langle\varphi\left(B^{q+r}\right) y, y\right\rangle\left\langle\phi\left(A^{p+r}\right) x, x\right\rangle+\left\langle\varphi\left(A^{q+r}\right) x, x\right\rangle\left\langle\phi\left(B^{p+r}\right) y, y\right\rangle .
\end{aligned}
$$

If $p>r>q>0$, then $f$ and $g$ are $t^{r}$-asynchronous and thus the reverse inequality holds.

(2) If $f(s)=s^{p}$ and $g(s)=\log s(s>1)$, then $f$ and $g$ are $t^{r}$-synchronous for all $p<r<0$ we have

$$
\begin{aligned}
& \left\langle\phi\left(B^{2 r}\right) y, y\right\rangle\left\langle\varphi\left(A^{p} \log (A)\right) x, x\right\rangle+\left\langle\varphi\left(A^{2 r}\right) x, x\right\rangle\left\langle\phi\left(B^{p} \log (B)\right) y, y\right\rangle \\
& \geq\left\langle\varphi\left(B^{r} \log (B)\right) y, y\right\rangle\left\langle\phi\left(A^{p+r}\right) x, x\right\rangle+\langle\varphi(A \log (A)) x, x\rangle\left\langle\phi\left(B^{p+r}\right) y, y\right\rangle .
\end{aligned}
$$


If $r<p<0$, then $f$ and $g$ are $t^{r}$-asynchronous and thus the reverse inequality holds.

(3) If $f(s)=\exp (s)=g(s)$, then $f$ and $g$ are $t^{r}$-synchronous for all $r \in \mathbb{R}$, so that we have

$$
\begin{gathered}
\left\langle\phi\left(B^{2 r}\right) y, y\right\rangle\langle\varphi(\exp (2 A)) x, x\rangle+\left\langle\varphi\left(A^{2 r}\right) x, x\right\rangle\langle\phi(\exp (2 B)) y, y\rangle \\
\geq 2\left\langle\varphi\left(A^{r} \exp (A)\right) x, x\right\rangle\left\langle\phi\left(B^{r} \exp (B)\right) y, y\right\rangle .
\end{gathered}
$$

Therefore, by choosing an appropriate function $h$ such that the assumptions in Remark 2.7 are fulfilled then one may generate family of inequalities from (2.1).

Corollary 2.8. Let $A$ be a selfadjoint operator with $\operatorname{sp}(A) \subset[\gamma, \Gamma]$ for some real numbers $\gamma, \Gamma$ with $0<\gamma<\Gamma$. If $f:[\gamma, \Gamma] \rightarrow \mathbb{R}$ is continuous and $f$ is t-synchronous on $[\gamma, \Gamma]$, then

$$
\left\langle\varphi\left(A^{2}\right) x, x\right\rangle \cdot\left\langle\varphi\left(f^{2}(A)\right) x, x\right\rangle \geq\langle\varphi(A f(A)) x, x\rangle^{2}
$$

for each $x \in H$ with $\|x\|=1$.

Proof. Setting $f=g, \phi=\varphi, B=A$ and $y=x$ in Corollary 2.5 we get the desired result.

Corollary 2.9. Let $A$ be a selfadjoint operator with $\operatorname{sp}(A) \subset[\gamma, \Gamma]$ for some real numbers $\gamma, \Gamma$ with $\gamma<\Gamma$. Let $h:[\gamma, \Gamma] \rightarrow \mathbb{R}$ be a non-negative continuous. If $f:[\gamma, \Gamma] \rightarrow \mathbb{R}$ is continuous and h-synchronous, then

$$
\begin{aligned}
\left\langle\phi\left(h^{2}(B)\right) y, y\right\rangle \cdot\langle\varphi(f(A)) x, x\rangle & +\left\langle\varphi\left(h^{2}(A)\right) x, x\right\rangle \cdot\langle\phi(f(B)) y, y\rangle \\
\geq\langle\varphi(h(A) f(A)) x, x\rangle \cdot\langle\phi(h(B)) y, y\rangle & +\langle\varphi(h(A)) x, x\rangle \cdot\langle\phi(h(B) f(B)) y, y\rangle
\end{aligned}
$$

for each $x \in H$ with $\|x\|=1$. In particular, we have

$$
\begin{gathered}
\left\langle\phi\left(h^{2}\left(A^{-1}\right)\right) x, x\right\rangle \cdot\langle\varphi(f(A)) x, x\rangle+\left\langle\varphi\left(h^{2}(A)\right) x, x\right\rangle \cdot\left\langle\phi\left(f\left(A^{-1}\right)\right) x, x\right\rangle \\
\geq\langle\varphi(h(A) f(A)) x, x\rangle \cdot\left\langle\phi\left(h\left(A^{-1}\right)\right) x, x\right\rangle \\
+\langle\varphi(h(A)) x, x\rangle \cdot\left\langle\phi\left(h\left(A^{-1}\right) f\left(A^{-1}\right)\right) x, x\right\rangle \quad(2.1
\end{gathered}
$$

Proof. Setting $g=1$ in (2.1) we get the first inequality (2.9). The second inequality holds by setting $B=A^{-1}$ and $y=x$ in (2.9).

Theorem 2.10. Let $A$ be a selfadjoint operator with $\operatorname{sp}(A) \subset[\gamma, \Gamma]$ for some real numbers $\gamma, \Gamma$ with $\gamma<\Gamma$. Let $h:[\gamma, \Gamma] \rightarrow \mathbb{R}$ be a non-negative continuous. If $f, g:[\gamma, \Gamma] \rightarrow \mathbb{R}$ are continuous and both $f$ and $g$ are $h$-synchronous ( $h$ asynchronous) on $[\gamma, \Gamma]$, then

$$
\begin{aligned}
\left\langle\phi\left(h^{2}(B)\right) y, y\right\rangle \cdot & f(\langle\varphi(A) x, x\rangle) g(\langle\varphi(A) x, x\rangle) \\
& +h^{2}(\langle\varphi(A) x, x\rangle) \cdot\langle\phi(f(B) g(B)) y, y\rangle \\
\geq(\leq)\langle\phi & (h(B) g(B)) y, y\rangle f(\langle\varphi(A) x, x\rangle) h(\langle\varphi(A) x, x\rangle) \\
+ & \langle\phi(f(B) h(B)) y, y\rangle h(\langle\varphi(A) x, x\rangle) g(\langle\varphi(A) x, x\rangle)
\end{aligned}
$$


for any $x \in K$ with $\|x\|=\|y\|=1$.

Proof. Since $\gamma 1_{H} \leq\langle A x, x\rangle \leq \Gamma 1_{H}$ then by employing $\varphi$, we get $\gamma 1_{K} \leq \varphi(A) \leq$ $\Gamma 1_{K}$. So that $\gamma \leq\langle\varphi(A) x, x\rangle \leq \Gamma$ for any $x \in K$ with $\|x\|=1$. Since $f, g$ are synchronous

$$
\begin{aligned}
{[(h(\langle\varphi(A) x, x\rangle) f(t)} & -h(t) f(\langle\varphi(A) x, x\rangle)] \\
& \times[h(\langle\varphi(A) x, x\rangle) g(t)-h(t) g(\langle\varphi(A) x, x\rangle)] \geq 0
\end{aligned}
$$

for any $t \in[\gamma, \Gamma]$ for any $x \in K$ with $\|x\|=1$.

Simplyfying the terms we have

$$
\begin{aligned}
& h^{2}(t) f(\langle\varphi(A) x, x\rangle) g(\langle\varphi(A) x, x\rangle) \\
& +h^{2}(\langle\varphi(A) x, x\rangle) \cdot f(t) g(t) \\
& \geq h(t) g(t) f(\langle\varphi(A) x, x\rangle) h(\langle\varphi(A) x, x\rangle) \\
& \quad+f(t) h(t) h(\langle\varphi(A) x, x\rangle) g(\langle\varphi(A) x, x\rangle) .
\end{aligned}
$$

Fix $x \in K$ with $\|x\|=1$. By utilizing the continuous functional calculus for the operator $B$ we have by the property (1.1) for inequality (2.13) we have

$$
\begin{aligned}
& h^{2}(B) f(\langle\varphi(A) x, x\rangle) g(\langle\varphi(A) x, x\rangle) \\
&+ h^{2}(\langle\varphi(A) x, x\rangle) \cdot f(B) g(B) \\
& \geq h(B) g(B) f(\langle\varphi(A) x, x\rangle) h(\langle\varphi(A) x, x\rangle) \\
& \quad+f(B) h(B) h(\langle\varphi(A) x, x\rangle) g(\langle\varphi(A) x, x\rangle) .
\end{aligned}
$$

Taking the map $\phi$ in the inequality (2.14), we get

$$
\begin{aligned}
\phi\left(h^{2}(B)\right) f(\langle\varphi(A) x, & x\rangle) g(\langle\varphi(A) x, x\rangle) \\
& +h^{2}(\langle\varphi(A) x, x\rangle) \cdot \phi(f(B) g(B)) \\
\geq \phi(h(B) g(B)) f(\langle\varphi(A) x, x\rangle) h(\langle\varphi(A) x, x\rangle) & \\
& +\phi(f(B) h(B)) h(\langle\varphi(A) x, x\rangle) g(\langle\varphi(A) x, x\rangle) .
\end{aligned}
$$

for any bounded linear operator $B$ with $\operatorname{sp}(B) \subseteq[\gamma, \Gamma]$ and $y \in H$ with $\|y\|=1$.

So that we can write $(2.15)$ in the form

$$
\begin{aligned}
\left\langle\phi\left(h^{2}(B)\right) y, y\right\rangle f & (\langle\varphi(A) x, x\rangle) g(\langle\varphi(A) x, x\rangle) \\
& +h^{2}(\langle\varphi(A) x, x\rangle) \cdot\langle\phi(f(B) g(B)) y, y\rangle \\
\geq\langle\phi(h(B) g(B)) y, y\rangle f(\langle\varphi(A) x, x\rangle) h(\langle\varphi(A) x, x\rangle) & +\langle\phi(f(B) h(B)) y, y\rangle h(\langle\varphi(A) x, x\rangle) g(\langle\varphi(A) x, x\rangle) .
\end{aligned}
$$

for each $x, y \in K$ with $\|x\|=\|y\|=1$, which proves the inequality in (2.11). The reverse sense follows similarly, and the proof is completed. 
Remark 2.11. Taking $\phi=\varphi$ in (2.12) we get

$$
\begin{aligned}
\left\langle\varphi\left(h^{2}(B)\right) y, y\right\rangle \cdot & f(\langle\varphi(A) x, x\rangle) g(\langle\varphi(A) x, x\rangle) \\
& +h^{2}(\langle\varphi(A) x, x\rangle) \cdot\langle\varphi(f(B) g(B)) y, y\rangle \cdot \\
\geq(\leq)\langle\varphi(h(B) g(B)) y, y\rangle f(\langle\varphi(A) x, x\rangle) h(\langle\varphi(A) x, x\rangle) & +\langle\varphi(f(B) h(B)) y, y\rangle h(\langle\varphi(A) x, x\rangle) g(\langle\varphi(A) x, x\rangle) .
\end{aligned}
$$

Also, by setting $B=A$ in (2.12) we get

$$
\begin{aligned}
\left\langle\phi\left(h^{2}(A)\right) y, y\right\rangle \cdot f & (\langle\varphi(A) x, x\rangle) g(\langle\varphi(A) x, x\rangle) \\
& +h^{2}(\langle\varphi(A) x, x\rangle) \cdot\langle\phi(f(A) g(A)) y, y\rangle \\
\geq(\leq)\langle\phi(h(A) g(A)) y, y\rangle f(\langle\varphi(A) x, x\rangle) h(\langle\varphi(A) x, x\rangle) & +\langle\phi(f(A) h(A)) y, y\rangle h(\langle\varphi(A) x, x\rangle) g(\langle\varphi(A) x, x\rangle) .
\end{aligned}
$$

Corollary 2.12. Let $A$ be a selfadjoint operator with $\operatorname{sp}(A) \subset[\gamma, \Gamma]$ for some real numbers $\gamma, \Gamma$ with $\gamma<\Gamma$. Let $h:[\gamma, \Gamma] \rightarrow \mathbb{R}$ be a non-negative continuous. If $f:[\gamma, \Gamma] \rightarrow \mathbb{R}$ is continuous and h-synchronous on $[\gamma, \Gamma]$, then

$$
\begin{array}{r}
\left\langle\phi\left(h^{2}(B)\right) y, y\right\rangle \cdot f^{2}(\langle\varphi(A) x, x\rangle)+\left\langle\phi\left(f^{2}(B)\right) y, y\right\rangle \cdot h^{2}(\langle\varphi(A) x, x\rangle) \\
\geq(\leq) 2\langle\phi(h(B) f(B)) y, y\rangle f(\langle\varphi(A) x, x\rangle) h(\langle\varphi(A) x, x\rangle)
\end{array}
$$

for any $x \in K$ with $\|x\|=\|y\|=1$. In particular, we have

$$
\begin{aligned}
\left\langle\varphi\left(h^{2}(B)\right) y, y\right\rangle \cdot f^{2}(\langle\varphi(A) x, x\rangle)+\left\langle\varphi\left(f^{2}(B)\right) y, y\right\rangle \cdot h^{2}(\langle\varphi(A) x, x\rangle) \\
\\
\geq(\leq) 2\langle\varphi(h(B) f(B)) y, y\rangle f(\langle\varphi(A) x, x\rangle) h(\langle\varphi(A) x, x\rangle),
\end{aligned}
$$

also, we have

$$
\begin{aligned}
\left\langle\phi\left(h^{2}(A)\right) y, y\right\rangle \cdot f^{2}(\langle\varphi(A) x, x\rangle)+\left\langle\phi\left(f^{2}(A)\right) y, y\right\rangle \cdot h^{2}(\langle\varphi(A) x, x\rangle) \\
\geq(\leq) 2\langle\phi(h(A) f(A)) y, y\rangle f(\langle\varphi(A) x, x\rangle) h(\langle\varphi(A) x, x\rangle) .
\end{aligned}
$$

for any $x \in K$ with $\|x\|=\|y\|=1$.

Proof. Setting $f=g$ in (2.11), respectively, we get the required results.

Corollary 2.13. Let $A$ be a selfadjoint operator with $\operatorname{sp}(A) \subset[\gamma, \Gamma]$ for some real numbers $\gamma, \Gamma$ with $0<\gamma<\Gamma$. If $f:[\gamma, \Gamma] \rightarrow \mathbb{R}$ are continuous and $t$-synchronous on $[\gamma, \Gamma]$, then

$$
\begin{aligned}
\left\langle\phi\left(B^{2}\right) y, y\right\rangle \cdot f^{2}(\langle\varphi(A) x, x\rangle)+\left\langle\phi\left(f^{2}(B)\right) y, y\right\rangle \cdot\langle\varphi(A) x, x\rangle^{2} \\
\\
\geq(\leq) 2\langle\phi(B f(B)) y, y\rangle f(\langle\varphi(A) x, x\rangle)\langle\varphi(A) x, x\rangle
\end{aligned}
$$

for any $x \in H$ with $\|x\|=1$.

Proof. Setting $h(t)=t$ in (2.16), respectively, we get the required results.

Theorem 2.14. Let $A$ be a selfadjoint operator with $\operatorname{sp}(A) \subset[\gamma, \Gamma]$ for some real numbers $\gamma, \Gamma$ with $\gamma<\Gamma$. Let $h:[\gamma, \Gamma] \rightarrow \mathbb{R}_{+}$be a positive function on $[\gamma, \Gamma]$. If 
$f, g:[\gamma, \Gamma] \rightarrow \mathbb{R}_{+}$are both positve, convex and $h$-synchronous on $[\gamma, \Gamma]$, then

$$
\begin{aligned}
& h^{2}(\langle A x, x\rangle)\langle f(B) y, y\rangle \cdot\langle g(B) y, y\rangle+h^{2}(\langle B y, y\rangle)\langle f(A) x, x\rangle \cdot\langle g(A) x, x\rangle \\
\geq & h(\langle A x, x\rangle) h(\langle B y, y\rangle)[f(\langle B y, y\rangle) g(\langle A x, x\rangle)+f(\langle A x, x\rangle) g(\langle B y, y\rangle)]
\end{aligned}
$$

for any $x, y \in H$ with $\|x\|=\|y\|=1$.

Proof. Since $f, g$ are $h$-synchronous and $\gamma \leq\langle A x, x\rangle \leq \Gamma, \gamma \leq\langle B y, y\rangle \leq \Gamma$ for any $x, y \in H$ with $\|x\|=\|y\|=1$, we have

$$
\begin{aligned}
& (h(\langle A x, x\rangle) f(\langle B y, y\rangle)-h(\langle B y, y\rangle) f(\langle A x, x\rangle)) \\
& \quad \times(h(\langle A x, x\rangle) g(\langle B y, y\rangle)-h(\langle B y, y\rangle) g(\langle A x, x\rangle)) \geq 0
\end{aligned}
$$

for any $t \in[a, b]$ for any $x \in H$ with $\|x\|=1$.

Employing property (1.1) for inequality (2.19) we have

$$
\begin{aligned}
& h^{2}(\langle A x, x\rangle) f(\langle B y, y\rangle) g(\langle B y, y\rangle) \\
& +h^{2}(\langle B y, y\rangle) f(\langle A x, x\rangle) g(\langle A x, x\rangle) \\
& -h(\langle A x, x\rangle) h(\langle B y, y\rangle) f(\langle B y, y\rangle) g(\langle A x, x\rangle) \\
& -h(\langle B y, y\rangle) h(\langle A x, x\rangle) f(\langle A x, x\rangle) g(\langle B y, y\rangle) \geq 0
\end{aligned}
$$

for any bounded linear operator $B$ with $\operatorname{sp}(B) \subseteq[\gamma, \Gamma]$ and $y \in H$ with $\|y\|=1$.

Now, since $f$ and $g$ are convex then we have

$$
\begin{aligned}
& \quad h^{2}(\langle A x, x\rangle)\langle f(B) y, y\rangle \cdot\langle g(B) y, y\rangle+h^{2}(\langle B y, y\rangle)\langle f(A) x, x\rangle \cdot\langle g(A) x, x\rangle \\
& \quad \geq h^{2}(\langle A x, x\rangle) f(\langle B y, y\rangle) \cdot g(\langle B y, y\rangle)+h^{2}(\langle B y, y\rangle) f(\langle A x, x\rangle) \cdot g(\langle A x, x\rangle) \\
& \geq h(\langle A x, x\rangle) h(\langle B y, y\rangle)[f(\langle B y, y\rangle) g(\langle A x, x\rangle)+f(\langle A x, x\rangle) g(\langle B y, y\rangle)]
\end{aligned}
$$

for each $x, y \in H$ with $\|x\|=\|y\|=1$. Setting $B=A^{-1}$ and $y=x$ in (2.21) we get the required result in (2.18). The reverse sense follows similarly.

Theorem 2.15. Let $A$ be a selfadjoint operator with $\operatorname{sp}(A) \subset[\gamma, \Gamma]$ for some real numbers $\gamma, \Gamma$ with $\gamma<\Gamma$. Let $h:[\gamma, \Gamma] \rightarrow \mathbb{R}_{+}$be a positive function on $[\gamma, \Gamma]$. If $f, g:[\gamma, \Gamma] \rightarrow \mathbb{R}_{+}$are both positve, convex and $h$-synchronous on $[\gamma, \Gamma]$, then

$$
\begin{gathered}
h^{2}(\langle\varphi(A) x, x\rangle)\langle\phi(f(B)) y, y\rangle \cdot\langle\phi(g(B)) y, y\rangle \\
+h^{2}(\langle\phi(B) y, y\rangle)\langle\varphi(f(A)) x, x\rangle \cdot\langle\varphi(g(A)) x, x\rangle \\
\geq h^{2}(\langle\varphi(A) x, x\rangle) f(\langle\phi(B) y, y\rangle) \cdot g(\langle\phi(B) y, y\rangle) \\
+h^{2}(\langle\phi(B) y, y\rangle) f(\langle\varphi(A) x, x\rangle) \cdot g(\langle\varphi(A) x, x\rangle) \\
\geq h(\langle\varphi(A) x, x\rangle) h(\langle\phi(B) y, y\rangle) \times[f(\langle\phi(B) y, y\rangle) g(\langle\varphi(A) x, x\rangle) \\
+f(\langle\varphi(A) x, x\rangle) g(\langle\phi(B) y, y\rangle)]
\end{gathered}
$$

for any $x, y \in H$ with $\|x\|=\|y\|=1$.

Proof. Since $\gamma \cdot 1_{H} \leq A, B \leq \Gamma \cdot 1_{H}$ then $\gamma \cdot 1_{K} \leq \varphi(A) \leq \Gamma \cdot 1_{K}$ and $\gamma \cdot 1_{K} \leq \phi(B) \leq$ $\Gamma \cdot 1_{K}$. So that for any $x, y \in H$ with $\|x\|=\|y\|=1$, we have $\gamma \leq\langle\varphi(A) x, x\rangle \leq \Gamma$ 
and $\gamma \leq\langle\phi(B) y, y\rangle \leq \Gamma$

$$
\begin{aligned}
& (h(\langle\varphi(A) x, x\rangle) f(\langle\phi(B) y, y\rangle)-h(\langle\phi(B) y, y\rangle) f(\langle\varphi(A) x, x\rangle)) \\
& \times(h(\langle\varphi(A) x, x\rangle) g(\langle\phi(B) y, y\rangle)-h(\langle\phi(B) y, y\rangle) g(\langle\varphi(A) x, x\rangle)) \geq 0
\end{aligned}
$$

for any $t \in[a, b]$ for any $x \in H$ with $\|x\|=1$.

Employing property (1.1) for inequality (2.23) we have

$$
\begin{gathered}
h^{2}(\langle\varphi(A) x, x\rangle) f(\langle\phi(B) y, y\rangle) g(\langle\phi(B) y, y\rangle) \\
+h^{2}(\langle\phi(B) y, y\rangle) f(\langle\varphi(A) x, x\rangle) g(\langle\varphi(A) x, x\rangle) \\
-h(\langle\varphi(A) x, x\rangle) h(\langle\phi(B) y, y\rangle) f(\langle\phi(B) y, y\rangle) g(\langle\varphi(A) x, x\rangle) \\
-h(\langle\phi(B) y, y\rangle) h(\langle\varphi(A) x, x\rangle) f(\langle\varphi(A) x, x\rangle) g(\langle\phi(B) y, y\rangle) \geq 0 .
\end{gathered}
$$

Now, since $f$ and $g$ are postive convex functions then we have

$$
\begin{gathered}
h^{2}(\langle\varphi(A) x, x\rangle)\langle\phi(f(B)) y, y\rangle \cdot\langle\phi(g(B)) y, y\rangle \\
+h^{2}(\langle\phi(B) y, y\rangle)\langle\varphi(f(A)) x, x\rangle \cdot\langle\varphi(g(A)) x, x\rangle \\
\geq h^{2}(\langle\varphi(A) x, x\rangle) f(\langle\phi(B) y, y\rangle) \cdot g(\langle\phi(B) y, y\rangle) \\
+h^{2}(\langle\phi(B) y, y\rangle) f(\langle\varphi(A) x, x\rangle) \cdot g(\langle\varphi(A) x, x\rangle) \\
\geq h(\langle\varphi(A) x, x\rangle) h(\langle\phi(B) y, y\rangle) \times[f(\langle\phi(B) y, y\rangle) g(\langle\varphi(A) x, x\rangle) \\
+f(\langle\varphi(A) x, x\rangle) g(\langle\phi(B) y, y\rangle)]
\end{gathered}
$$

for each $x, y \in H$ with $\|x\|=\|y\|=1$, which proves the required result in (2.22). The reverse sense follows similarly.

\section{REFERENCES}

1. M.W. Alomari, On Pompeiu-Chebyshev functional and its generalization, Preprint (2017). Availiable at arXiv:1706.06250v2

2. M.W. Alomari, Pompeiu-Čebyšev type inequalities for selfadjoint operators in Hilbert spaces, Adv. Oper. Theory, 3 no. 3 (2018), 9-22.

3. S.S. Dragomir, Čebyšev's type inequalities for functions of selfadjoint operators in Hilbert spaces, Linear and Multilinear Algebra, 58 no 7-8 (2010), 805-814.

4. S.S. Dragomir, Operator inequalities of the Jensen, Čebyšev and Grüss type, Springer, New York, 2012.

5. T. Furuta, J. Mićić, J. Pečarić and Y. Seo, Mond-Pečarić method in operator inequalities. Inequalities for bounded selfadjoint operators on a Hilbert space, Element, Zagreb, 2005.

6. M.S. Moslehian and M. Bakherad, Chebyshev type inequalities for Hilbert space operators, J. Math. Anal. Appl. 420 (2014), no. 1, 737-749.

7. J.S. Matharu and M.S. Moslehian, Grüss inequality for some types of positive linear maps, J. Operator Theory 73 (2015), no. 1, 265-278.

Department of Mathematics, Faculty of Science and Information Technology, Irbid National University, P.O. Box 2600, Irbid, P.C. 21110, Jordan.

Email address: mwomath@gmail.com 